\title{
THE EVOLUTION OF THE MODERN INSTRUMENTS TOWARD VIRTUAL DISTRIBUTED ARCHITECTURES
}

\author{
Loredana Cristaldi, Alessandro Ferrero, Simona Salicone \\ Politecnico di Milano, Piazza Leonardo da Vinci 32 - 20133 Milano, \\ \{loredana.cristaldi,alessandro.ferrero,simona.salicone\}@polimi.it
}

\begin{abstract}
During the last decades, Digital Signal Processing techniques and devices have undergone an impressive evolution that led their performance to increase manifold. One of the fields that has greatly benefited of this evolution is that of instrumentation and measurement. DSP-based techniques are presently the most employed techniques in industrial and scientific measurement applications, so that modern instruments are actually computers with dedicated interfaces and dedicated software. The full exploitation of the computing features has led to the development of the Virtual Instruments, and, more recently, to the development of Distributed Instruments. This paper is aimed at briefly discussing the architecture of the modern Virtual, Distributed Instruments, some of their most significant applications and the major metrological problems raised by these systems.
\end{abstract}

Keywords: Virtual Instrument, Distributed Instruments, DSP techniques, Measurement uncertainty.

\section{INTRODUCTION}

One of the most impressive technical evolutions, during the last decades, has been, quite likely, that of Digital Signal Processing, in its broadest meaning: all devices involved in DSP applications, from the Analog-to-Digital (AD) and Digital-to-Analog (DA) Converters, to the storage and computing devices, have manifold increased their performances, and the mathematical theory of the discrete-time signals has developed as well by proposing new, faster algorithms for signal analysis in the different possible domains.

Several fields received great benefit from this evolution. One of them is, for sure, that of measurements and instrumentation. The major advantage carried by the DSP application to measurements was the capability of extracting the required measurement information from the signals in a straightforward way, without the need for any additional conversion into a different physical phenomenon (as it was the case of the old electromechanical instruments). A significant example of these benefits is given by the characterization of magnetic materials [1], for which the analog techniques required long and cumbersome procedures and showed little accuracy.

During the pioneering stage of the application of the DSP-techniques to measuring instruments, in the late ' 70 s, the only available computing devices were the microcomputers. These devices allowed one to implement only simple measurement algorithms, though the implementation itself was not simple at all, especially if real-time applications were needed: the algorithms had to be coded in assembler language, and the available hardware resources had to be fully exploited, thus requiring good skills in computer science to the measurement experts. Moreover, the way these new instruments were interfaced to the operator was totally different from that of the more traditional instruments, and this could potentially bewilder the operators.

When the first PCs burst into the technical and scientific community, with their user-friendly interface, some pioneering attempts to exploit these facilities in the development of Personal Instruments were made [2], opening the way to the far more effective architecture of the Virtual Instruments [3].

The turning point was reached when the modern computers overperformed the strict requirements of the DSP algorithms, and a significant amount of computational capability was still available after having executed the measurement algorithms. These available resources were used to develop a userfriendly graphical interface, so that the operators could access a virtual front panel absolutely similar to the traditional ones, to which they were used. Moreover, a graphical interface was also added for implementing the measurement algorithm, thus changing the development of DSP-based instruments from a difficult task for skilled programmers into a game-like graphical exercise. The Virtual Instrument 
(VI) have become a reality, and measuring instruments have evolved from a dedicated hardware structure to a general purpose computer structure, equipped with dedicated interfaces (the AD converters) and dedicated software.

This structure can now fully exploit the most recent evolution of nowadays computers: their capability of being easily connected together into a network of cooperating resources. This means that any computer, on which a VI is running, can be connected to any other computer, on which another VI is running, and becomes a part of a larger cooperating system of Distributed Instruments.

The possible applications of this distributed architecture of instruments are practically countless. Some of them have been already implemented in different areas, from electric power quality measurements, monitoring and diagnostic systems, to environmental measurement obtained by means of a dedicated sensor network.

The hidden face of the moon is that these measurement systems pose a number of new metrological issues when we try to answer the basic question of the measurement science: how accurate is the measurement result we get from these measuring architectures?

This paper, after shortly discussing the architecture of the modern Virtual, Distributed Instruments, will show some significant applications and will discuss the major metrological problems met in the characterization of these systems.

\section{VI STRUCTURE}

A modern measurement system, based on DSP techniques and structured according to a VI architecture, can be represented [4] by the block diagram shown in Fig. 1. The following main blocks can be identified.

- Transducer and Signal Conditioning (T\&C) block, that adapts the input signals to the subsequent $\mathrm{AD}$ conversion block and pre-filters the input signals to avoid possible aliasing effects.

- The AD conversion unit (ADC), that converts the input, continuous-time, analog signals into discrete-time signals, with the resolution and sampling rate required by the characteristic of the input signals to ensure a correct execution of the measurement algorithm.

- The storage memory (MEM), where the samples of the discrete-time signals are stored, after the $\mathrm{AD}$ conversion stage, for further processing.

- The computing unit (Comp), that processes the stored samples of the discrete-time signals in order to execute the measurement algorithm and provide the required measurement result.

The four blocks shortly described above define

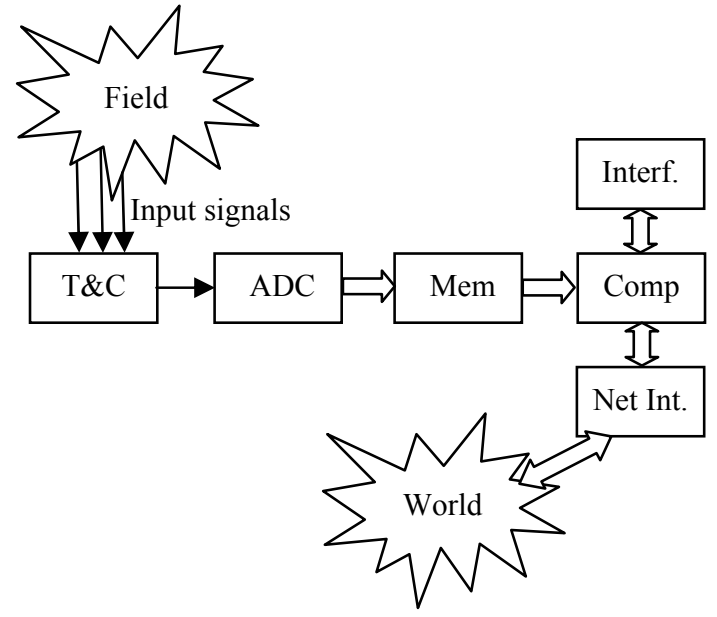

Fig. 1 - Block diagram of a modern DSPbased instrument: $T \& C$ : Transducer and signal conditioning; ADC: AD conversion unit; Mem: storage memory; Comp: DSP device; Interf: man-machine interface; Net Int.: interface to the network

the architecture of a generic DSP-based instrument, within which any measurement process can be seen as one that extracts the desired information from the input signals by means of DSP-techniques [4].

The innovation that this architecture brought into the measurement science and practice was the drastic modification in the traditional measurement paradigm, that associates a single, dedicated measuring device to each measurement process. On the other hand, with DSP-based systems, a measurement process is associated with a dedicated algorithm implemented on the same general purpose architecture described by the four blocks above recalled. Therefore, the same hardware structure can perform totally different measurement procedures by simply uploading a different program.

The major drawback of these architectures, when they were first introduced, was the unfriendly manmachine interface, with limited or nil graphical facilities, and the need of good programming skills to implement the measurement algorithms.

If the computational power of the computing unit is not fully exploited by the measurement algorithm, and some resources are left to run other concurrent tasks, the general purpose architecture described by the aforementioned four blocks can be specialized into a VI architecture [4] if a dedicated man-machine interface (Interf block in Fig. 1) is added, whose task is that of providing a user-friendly front panel and a user-friendly programming interface.

In particular, a user-friendly front panel is expected to provide a graphic representation, by means of dedicated icons, of the conventional controls (buttons, selectors, knobs, ...) and indicators (numerical displays, gauges, scope-like displays, leds, $\ldots)$, that are familiar to the operator and can be virtually operated in the same way as the real controls 
and indicators.

Moreover, a great flexibility is offered to the VI designer, since dedicated front panels can be designed, merging, partially or totally, the functionality of different traditional instruments to implement the desired measuring features in a very efficient way: only the controls and indicators that are necessary for the required function are employed, and the operator attention is not distracted by unnecessary controls and indicators. Fig. 2 shows an example of front panel, for a VI dedicated to detect transient disturbances on the supply voltages of an electric customer [5].

As for the programming interface, the full exploitation of the graphical programming techniques has led to an impressive simplification of the definition of the measurement procedures by means of object-oriented, data-driven techniques [4].

The main advantage of this programming technique is that it allows the programmer to describe the measurement procedure without any need for a conventional procedural programming language, that looks generally rather obscure to people that are not computer scientists and engineers. On the other hand, visual programming allows one to represent data and operations by icons, so that the measure-

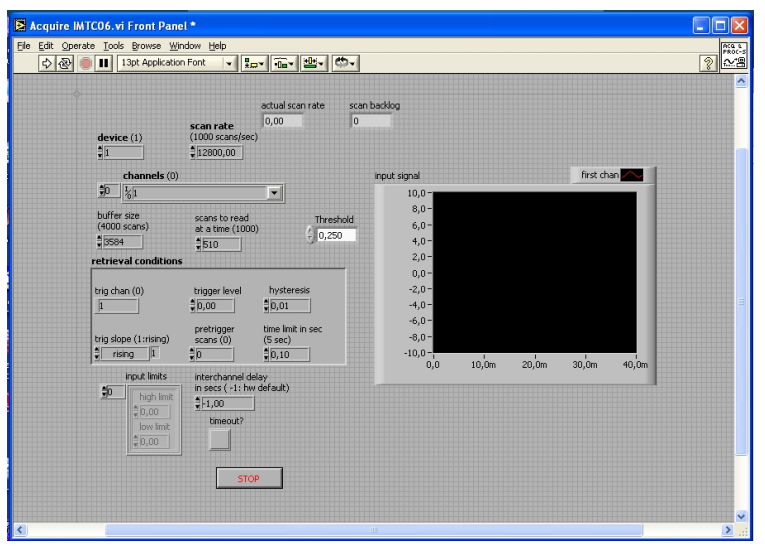

a)

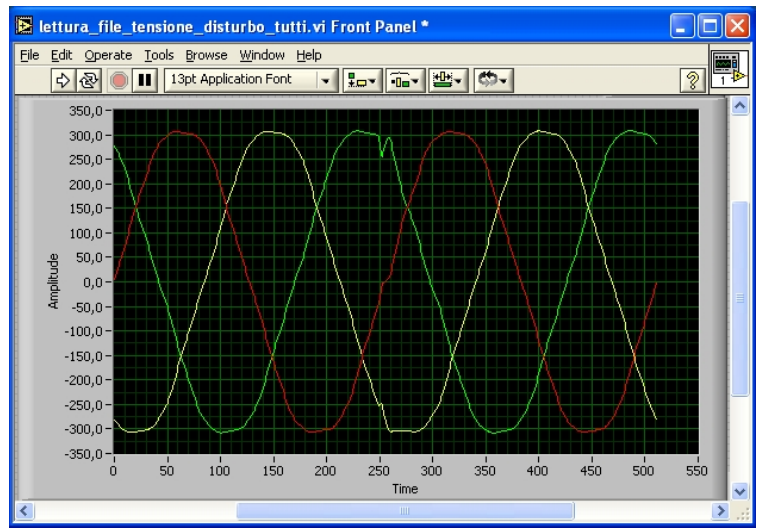

b)

Fig. 2 - a): Example of front panel of a VI for detecting transient disturbances of the supply voltages of an electric customer; b) detected disturbance ment procedure is described by drawing a procedural diagram (the block diagram) that defines data dependencies and operations to be performed. The nodes of the diagram represent high-level operations, such as DSP blocks, visual data presentation, peripheral management, .... Each node is visually represented by a dedicated icon, showing input and output connections that allow receiving and delivering operands and results, respectively. Directed paths represent the data dependencies among operations [4].

Modern graphic languages for developing VIs offer incredibly huge libraries of high-level functional blocks, so that implementing a VI really looks like a game-like graphical exercise. The most interesting feature, from the point of view of the measurement expert, is that the measurement procedure graph drawn using a graphic language coincides with the conventional block diagram that the measurement engineer is accustomed to draw to describe the overall measurement procedure, as well as the signal paths inside the instrument. Therefore, any measurement expert can design a dedicated VI with a very limited extra training, since the conventional block diagram, drawn by means of a proper graphical interface, is automatically translated into the program implementing the VI itself. Fig. 3 shows a particular of the block diagram of the VI whose front panel is shown in Fig. 2.

As already mentioned, a VI architecture such as the one described here can be seen as a computer equipped with dedicated hardware peripherals and dedicated software. If we now think of the modern computers, we immediately realize that it is impossible to think of nowadays computers without thinking them connected to a WAN, such as Internet. Almost every computer is equipped with an interface to the network and with dedicated routines that connect it to other computers over the network in a simple and straightforward way. In this vision, each single computer can be seen as the single unit of a network of units that are sharing resources and

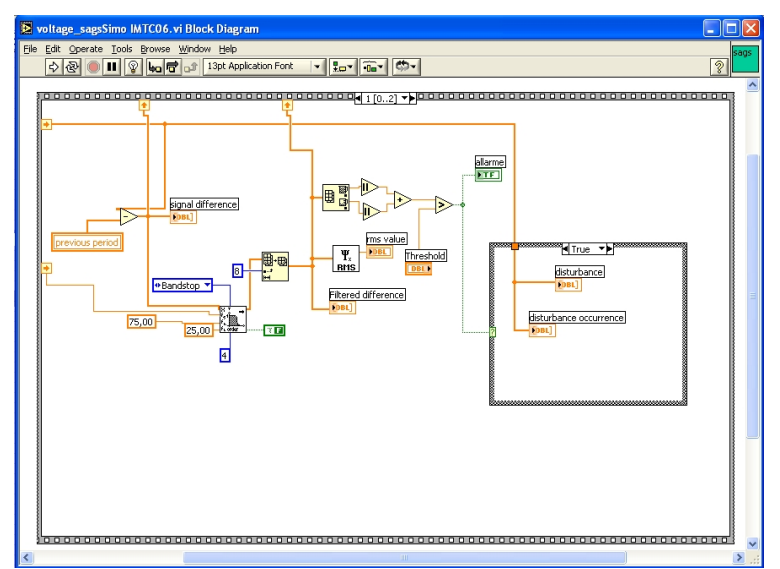

Fig. 3 - Block diagram for the disturbance detection algorithm of the VI shown in Fig. 2. 
cooperating to find the best solution to a given problem.

This same paradigm can be applied to the modern VIs that, once equipped with an interface to a LAN or WAN (block Net Int. in Fig. 1), can be connected to other VIs and realize a network of sensing units that share their resources and cooperate to implement a measurement procedure.

Distributed Instruments are hence obtained and represent the last frontier of the modern instruments. As it will be shown in the next sections, they can be employed to implement several measurement procedures, ranging from a modern reinterpretation of remote measurements, where a remote unit broadcasts its measurement results to a central unit over Internet, to more complex applications, such as remote calibrations or environmental monitoring by means of hundreds or thousands of smart sensors spread over a wide area, connected together and to a central unit that collects the measured data of all monitored quantities.

\section{SOME APPLICATIONS}

The number of applications of the modern distributed measurement systems is virtually infinite, and steadily growing as the employed technology evolves and makes new applications technically feasible and cost effective. The following examples cannot, therefore, represent an exhaustive list of the possible applications: they are only aimed at giving the reader a flavor of some of the most significant possible applications and also the new metrological problems that these systems have brought into light.

\subsection{REMOTE MEASUREMENT SYSTEMS}

Remote measurement systems have been extensively used for a long time, mainly to monitor large plants, such as electricity, water and gas distribution networks. Measured data were generally broadcasted on a private network, or on a dedicated line of a public network (such as telephone lines or radio channels). The measured data could be accessed only from the central unit to which data were broadcasted.

The main advantage of network connected instruments is that measured data can be accessed form virtually any point connected to the network, provided the necessary authorization is granted to the user. Therefore, the measured data are not confined to a single central location, but are available from any location. As far as large plant monitoring is concerned, the main advantage is that there is no need to guard the system from a definite location: the person on duty to monitor the system and take all needed action in case of alarm can do this even from home, for instance during the night or holidays.
When the measured data are related to quantities of public interest, such as meteo-related quantities (temperature, humidity, wind speed, etc.) they can be made accessible to public in a quite immediate way, by posting them on a dedicated web page. Similarly, data measured in large scientific experiments (such as those concerned with the physics of the elementary particles) can be accessed by scientists from everywhere around the world, without the need for being physically present to the experiment.

The most interesting evolution of the remote measurement systems is probably the implementation of remote laboratories for didactic purposes. E.learning is one of the keywords of the modern education system, since it offers the possibility of spreading knowledge also where the potential students cannot attend, for any kind of reasons, traditional classes.

Instrumentation and measurement is a very peculiar subject, because it cannot be fully understood if students cannot practice the studied concepts during lab classes. Virtual, distributed instruments do represent the solution to this problem, since different experiments can be implemented in different locations, gathered in a unique virtual lab through a suitable portal, and made accessible to students through a simple browser. Several applications are already available [6-9], and some of them are now currently used $[8,9]$.

\subsection{DIAGNOSTIC TOOLS FOR COMPLEX SYSTEMS}

Diagnostic systems can be seen as an evolution of the monitoring systems. In fact, whenever the monitoring system detects deviations, in the measurement quantities, from the optimal values, it can activate, locally or centrally, dedicated diagnostic routines, capable of detecting anomalies in the behavior of the single components before they evolve into faults [10].

In complex systems, the diagnosis issue becomes far more critical, since potential problems might be caused not only by anomalies or latent faults in the single components, but also in possible incompatibilities between a single component and the rest of the system. Of course, it would be advisable to detect all possible problems before installing the component and operate it.

Simulation tools are quite helpful, and several advanced tools are now available for simulating very large and complex systems [11]. However, unpredictable problems are generally unveiled by simulation, and can be discovered only by experimental tests.

A good intermediate solution is to include experimental data inside the simulation, according to 
the so-called "hardware-in-the-loop" solution [11]. If measurement results can be broadcasted and the measurement set-up can be changed from a remote location, a real component can be operated remotely, for instance at the manufacturer site, with the test conditions given by the simulator of the whole system, and the data measured on the real components can be fed back to the simulator, to check whether it may create problems to the whole system or not [12]. The advantage, especially economical, of this solution is quite evident.

\subsection{REMOTE CALIBRATION}

The periodical calibration of the instruments is not only recommended by the good practice of measurement, but is also imposed by several national and international Standards. The most important recommendation is the one included in the ISO Standards of the 9000 series, that require the periodical calibration, performed by an accredited laboratory, of all instruments employed in the production process monitored by the quality procedures.

This periodical calibration has generally a nonnegligible cost, not only because of the calibration fee paid to the calibration lab, but mainly because of the cost of not having the instrument available for the whole period required by the calibration, including the shipping time of the instrument to and from the calibration lab.

A significant cost reduction would be possible if the calibration laboratories could ship a traveling standard to the customer that had required a calibration and both the traveling standard and the instrument to be calibrated were accessible over Internet.

Prototypes of the traveling standards [13] and the VIs for accessing it and the instrument under test $[14,15]$ are already available. The most critical part of this whole process is, of course, data security, since the process must be always under control of the calibration laboratory, and the operations performed by the customer must be limited to a minimum: the connection of the traveling standard to the instrument to be calibrated, the connection of both them, through a standard interface, to a PC connected to Internet, and the connection, through a browser, to a dedicated web page at the calibration lab site [14].

A possible solution to the security issue is the use of the mobile, multi agent technique $[14,15]$. In this way, the routines that control the traveling standard and the instrument to be calibrated, and read the measured values are uploaded on the local PC directly from the lab site, and no local operation is required at the customer site. In other words, the calibration lab gains full control of every operation performed on the instrument under calibration, and the calibration procedure is protected against any malicious attack.

\subsection{NETWORK OF COOPERATING IN- STRUMENTS}

The most intriguing evolution of the Distributed Instruments leads to the networks of cooperating instruments.

The idea of such networks comes from the need to monitor very large and complex systems, from the natural ones, such as the environment, to the artificial, man-originated ones, such as, for instance, the electric grid.

The ultimate monitoring goal is the real-time estimation of the system status, so that anomalous, dangerous evolutions can be anticipated and, possibly, avoided. This requires the simultaneous analysis of physical quantities in virtually every node of the system. Moreover, the optimal representation of the system, under the different conditions, may require different measurements. Therefore, the employed instruments must be capable of exchanging information among each other and adapting their measurement procedures to the modified system conditions.

Two applications can be considered, as significant examples of cooperating instruments: electric power quality monitoring and environmental monitoring.

In nowadays electric systems, due to the ever increasing diffusion of electronic non-linear and timevariant loads and the diffusion of small generating units (mainly small photo-voltaic and wind-turbine generators), periodic and transient disturbances are injected in the network, so that the quality of the voltages supplied by the Utilities and the quality of the currents consumed by the customers are rapidly deteriorating.

The only effective way to detect the injected disturbances and identify the source injecting these disturbances is the simultaneous measurement of several quantities and indices on all lines supplying and leaving the network nodes, and process them to quantify how far each single line contributes to power quality deterioration $[16,17]$.

This result can be attained if the instruments employed to measure the quantity and indices related to each single line can communicate with each other, synchronize the measurement periods and broadcast the measurement results to the post-processing unit for the determination of the final power-quality indices [16].

Environmental monitoring is among nowadays priorities of the developed Countries, to ensure a sustainable development with a reduced impact on the environment, and monitor the evolution of natural phenomena to warn the population against the 
possible risks of calamities.

This task requires a large number of sensing unites, dispersed over the monitored area, that communicate with each other and a central unit that collects and processes the incoming data [18-20].

Due to the generally broad extension of the area to be monitored, a number of new problems must be solved, concerned, for instance, with the low energy consumption required for each unit, the automatic search for the best path for transmitting the measured data, the automatic reassignment of the tasks of a faulty unit to the safe ones with a minimum downgrade of the overall network performance.

The solution of these problems is the present frontier of the modern Distributed Instruments [1820] and requires great skills in both measurement and computer science.

\section{METROLOGY ISSUES}

According to the previous sections, implementing a VI and networking different VIs to form a distributed system is a relatively simple task, due to the availability of user-friendly interfaces for both creating a very efficient front panel and programming the measurement procedure, and the availability of very large libraries of measurement functions based on DSP techniques.

Very often, this relative ease leads to the apparent conclusion that implementing a VI does not require any particular knowledge of the mathematics of the discrete-time signals and systems. This is a very dangerous conclusion, since the mathematics of the discrete-time signals and the implications of the sampling theorem are quite complex and not immediate to understand. The same sampling strategy may lead either to correct results or to gross mistakes for almost negligible changes in the measurement conditions. The consequences of the spectral leakage may be as dramatic as those of the aliasing [21], though they are often not considered by several measurement practitioners that have only a superficial knowledge of the mathematics of the discretetime signals.

Moreover, the relative ease of implementing any measurement procedure also hides the complexity of evaluating the measurement uncertainty. It is well known that uncertainty expression and estimation is the most important task in metrology, since a measurement result is totally useless if it is provided without its associated uncertainty [22].

When the measurement result is obtained by means of a complex processing of a large number of samples, the final measurement result can be considered as the result of an indirect measurement, where each sample represents each directly measured quantity. The evaluation of the uncertainty associ- ated to the final result, starting from the estimated uncertainty values for each single sample, is not an immediate task, both from a theoretical and a practical point of view.

Moreover, the present reference Standard [22] has been worked out when the DSP techniques were at a very early stage of development, and did not consider the problems posed by the digital instruments. Trying to apply its recommendations to the modern, complex systems may result in a cumbersome and unreliable procedure.

A more general mathematical approach to the problem than the one suggested by [22] has been recently proposed, and seems to be quite promising $[23,24]$. However, the problem of the metrological characterization of the complex VIs is far from being solved and must be carefully considered by developers and users of such systems.

Distributed measurement systems pose an additional problem, that may become critical when simultaneous measurements are required in the different locations of the single measurement units of the distributed system: the synchronization of the different units in the system.

A good synchronization of the different clocks can be obtained using a GPS signal. However, this does not prevent the possible problems coming by the net latencies if data are transmitted over a public network, such as Internet. Once again, the most critical problem is how to estimate the contribution given by the possible latencies to the final measurement uncertainty. Although the problem has been addressed, we are still far from a satisfying universal solution [25].

\section{CONCLUSION}

The above sections have provided a brief survey of the main characteristics of the modern Virtual, Distributed Instruments and some significant examples of their possible applications.

It can be concluded that these measuring systems combine the most important advantages offered by the DSP techniques and AD conversion devices, and the modern visual programming techniques, thus virtually changing any computer into a powerful measuring instrument.

The main feature of these measuring systems is the relative ease with which complex measurement procedures can be implemented, the system can be operated and the results displayed or transmitted to a remote location for further processing.

The main problem posed by these same systems is their metrological characterization. Uncertainty estimation, though mandatory, is still far from being a known, universally accepted task. A lot of work is still required to the experts to find a suitable, theo- 
retically and practically sound solution to this problem.

In conclusion, the modern virtual, distributed instruments provide a technically and economically viable solution to several measurement problems that appeared to be unsolvable only a few years ago. Their only drawback is that, until the metrology issues addressed in the previous section 4 are not solved, the more complex the measurement procedures become, the less we know about how accurate is the solution they provide. Being aware of this problem is a first step to avoid an incorrect use of the measurement results.

\section{REFERENCES}

[1] E. Carminati, A. Ferrero. A virtual instrument for the measurement of the characteristic of magnetic materials. IEEE Trans. Instr. Meas., vol. 41, no. 6, 1992, p. 1005-1009.

[2] A. Ferrero. Software for personal instruments. IEEE Trans. Instr. Meas., vol. 39, no. 6, 1990, p. 860-863.

[3] H. J. W. Spoelder. Virtual Instrumentation and Virtual Environment. IEEE Instrumentation and Measurement Magazine, Vol. 2, no. 3, 1999, p. 14-19.

[4] L. Cristaldi, A. Ferrero, V. Piuri. Programmable Instruments, Virtual Instruments and Distributed Measurement Systems: what is really useful, innovative and technically sound? IEEE Instrumentation and Measurement Magazine, Vol. 2, no. 3, 1999, p. 20-27.

[5] A. Ferrero, S. Salicone. An easy VI program to detect transient disturbances in the supply voltage. IEEE Trans. Instr. Meas., vol. 54, no. 4, 2005, p. 349-356.

[6] L. Benetazzo, M. Bertocco, F. Ferraris, A. Ferrero, C. Offelli, M. Parvis, V. Piuri. A Webbased distributed virtual educational laboratory. IEEE Trans. Instr. Meas., vol. 49, no. 2, 2000, p. 1471-1474.

[7] A. Ferrero, S. Salicone, C. Bonora, M. Parmigiani. ReMLab: a Java-based remote, didactic measurement laboratory. IEEE Trans. Instr. Meas., vol. 52, no. 3, 2003, p. 710-715.

[8] G. Andria et al. Remote Didactic Laboratory "G. Savastano": the Italian Experience for the E-learning at the Technical Universities in the Field of the Electrical and Electronic Measurements, Architecture and Delivered Services. Proceedings of the IEEE Instrumentation and Measurement Technology Conference - IMTC, 2006, pp. 998-1002.

[9] G. Andria et al. Remote Didactic Laboratory "G. Savastano": the Italian Experience for the E-learning at the Technical Universities in the
Field of the Electrical and Electronic Measurements, Overview on Didactic Experiments Proceedings of the IEEE Instrumentation and Measurement Technology Conference - IMTC, 2006, pp. 1537-1542.

[10] L. Cristaldi, A. Ferrero, A. Monti, S. Salicone. A versatile monitoring system for AC motor drives, Proceedings of the $3^{\text {rd }}$ IEEE Int. Symposium on Diagnostic for Electrical Machines, Power Electronics and Drives, September 2001, pp. 1-6.

[11] R. A. Dougal, A. Monti. The Virtual Test Bed As A Tool for Rapid System Engineering. Proceedings of the $1^{\text {st }}$ Annual IEEE Systems Confrence, 9-13 April 2007, pp. $1-6$.

[12] L. Cristaldi, A. Ferrero, A. Monti, F. Ponci, W. McKay, R. A. Dougal. A virtual environment for remote testing of complex systems. IEEE Trans. Instr. Meas., vol. 54, no. 1, 2005, p. 123133.

[13] A. Carullo, M. Parvis, A. Vallan. A traveling standard for the calibration of data acquisition boards. IEEE Trans. Instr. Meas., vol. 53, no. 2, 2004, p. 557-560.

[14] M. Albu, A. Ferrero, F. Mihai, S. Salicone. Remote calibration using mobile, multiagent technology. IEEE Trans. Instr. Meas., vol. 54, no. 1, 2005, p. 24-30.

[15] S. De Capitani di Vimercate, A. Ferrero, M. Lazzaroni. Mobile Agent Technology for Remote Measurements. IEEE Trans. Instr. Meas., vol. 55, no. 5, 2006, p. 1559-1565.

[16] L. Cristaldi, A. Ferrero, S. Salicone. A distributed system for electric power quality measurement. IEEE Trans. Instr. Meas., vol. 51, no. 4, 2002, p. 776-781.

[17] C. Muscas, L. Peretto, S. Sulis. R. Tinarelli. Investigation on Multipoint Measurement Techniques for PQ Monitoring. IEEE Trans. Instr. Meas., vol. 55, no. 5, 2006, p. 311-315.

[18] A. M. Mielke, S. M. Brennan, M. C. Smith, D. C. Torney, A. B. Maccabe, M. J. F. Karlin. Independent sensor networks. IEEE Instrumentation and Measurement Magazine, Vol. 8, no. 2, 2005, p. 33-37.

[19] Md. A. Rahman, Md. S. Miah, W. Gueaieb, A. E. Saddik. SENORA: A P2P Service-Oriented Framework for Collaborative Multirobot Sensor Networks. IEEE Sensors Journal, vol. 7, no. 5, 2007, pp. 658-666.

[20] D. Potter. Smart plug and play sensors. IEEE Instrumentation and Measurement Magazine, Vol. 5, no. 1, 2002, p. 28-30.

[21] G. D'Antona, A. Ferrero. Digital Signal Processing for Measurement Systems. Theory and Applications. Springer. New York, NY, USA, 2006. 
[22] BIPM, IEC, IFCC, ISO, IUPAC, OIML, Guide to the Expression of Uncertainty in Measurement, 1993.

[23] S. Salicone. Measurement Uncertainty: an approach via the mathematical theory of evidence. Springer. New York, NY, USA, 2007.

[24] A. Ferrero, S. Salicone. Modeling and Processing Measurement Uncertainty Within the Theory of Evidence: Mathematics of RandomFuzzy Variables. IEEE Trans. Instr. Meas., vol. 56, no. 3, 2007, p. 704-716.

[25] L. Cristaldi, A. Ferrero, C. Muscas, S. Salicone, R. Tinarelli. The impact of Internet transmission on the uncertainty in the electric power quality estimation by means of a distributed measurement system. IEEE Trans. Instr. Meas., vol. 52, no. 4, 2003, p. 1073-1078.

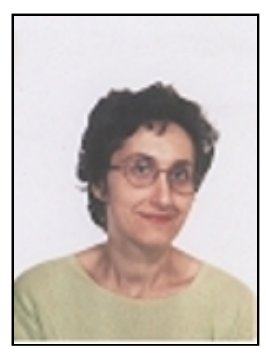

Loredana Cristaldi, was born in Catania, Italy. She received the M.Sc. degree in electrical engineering from the University of Catania, Catania, in 1992 and the Ph.D. degree in electrical engineering in 1996 from the Politecnico di Milano, Milano, Italy.

In 1999, she joined the Dipartimento di Elettrotecnica, Politecnico di Milano, as an Assistant Professor of electrical and electronic measurements. Since 2005 she has been an Associate Professor of electrical and electronic measurements at the same Department. Her research interests are in the field of the measurements of electric quantities under nonsinusoidal conditions, the characterization of magnetic materials, virtual instruments, measurement methods for reliability assessment and measurement methods for diagnosis of components and systems .

She is a member of the Italian Association for Electrical and Electronic Measurements (GMEE).

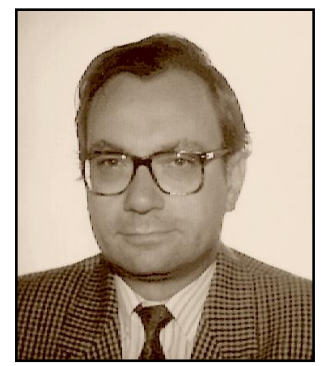

Alessandro Ferrero was born in Milano, Italy, in 1954. He received the M.S. degree in Electrical Engineering from the "Politecnico di Milano", Milano, in 1978. In 1983 he joined the Dipartimento di Elettrotecnica of the Politecnico di Milano as an Assistant Professor on Electrical Measurements. From 1987 to 1991 he was Associate Professor of "Measurements on Electrical Machines and
Systems" at the University of Catania, Catania, Italy. From 1991 to 1994 he was Associate Professor of Electrical Measurements at the Dipartimento di Elettrotecnica of the "Politecnico di Milano" University, Milano, Italy, where he has been Full Professor of Electrical and Electronic Measurements since 1994. His current research interests include the application of digital methods to electrical measurements, measurements on electric power systems under nonsinusoidal conditions and innovative methods for uncertainty estimation.

Prof. Ferrero has chaired the Italian Association on Electrical and Electronic Measurements (GMEE) for the three-year term 2004-2007 and is a member of the AdCom of the IEEE Instrumentation and Measurement Society. $\mathrm{He}$ is the recipient of the 2006 Joseph F. Keithley IEEE Field Award for Instrumentation and Measurement.

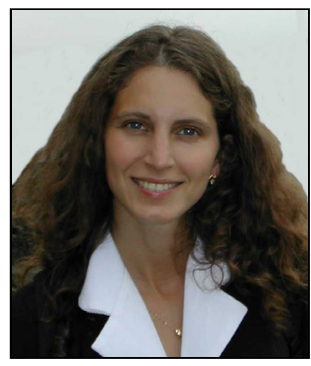

Simona Salicone was born in Milan, Italy, and received her M.Sc. degree in electrical engineering from the Politecnico di Milano, Milan, in 2000, and her Ph.D degree in electrical engineering from the same university, in 2004.

In 2000 she joined the Dipartimento di Elettrotecnica of the Politecnico di Milano as a part-time researcher on a research project aimed at the metrological characterization of complex, distributed measurement systems.

Since 2005 she has been an Assistant Professor of Electrical and Electronic Measurements at the same university. Her research interests are concerned with the analysis of advanced mathematical methods for uncertainty representation and estimation.

She is a member of the Italian Association for Electrical and Electronic Measurements (GMEE) and she is the 2005 recipient of the IEEE Instrumentation and Measurement Society Young Engineer Award. 